\title{
ALFABETIZACIÓN DE ADULTOS DESDE UNA PERSPECTIVA PSICOGENÉTICA ${ }^{1}$
}

\section{MARINA KRISCAUTZKY LAXAGUE}

Universidad Nacional Autónoma de México. Doctora en Ciencias con especialidad en investigación educativa, área de Psicolingüística, por el Departamento de Investigaciones Educativas del Centro de Investigación y Estudios Avanzados del Instituto Politécnico Nacional de México. Docente en la Facultad de Filosofía y Letras, Universidad Nacional Autónoma de México, y en la Universidad de La Plata, Argentina. ORCID 0000-0003-3489-1537. E-mail: mkriscau@unam.mx

\begin{abstract}
ADRIANA ARELI BRAVO LOZANO
Pedagoga egresada de la Facultad de Filosofía y letras de la Universidad Nacional Autónoma de México. Especialista en TIC para la educación por la Dirección General de Cómputo y de Tecnologías de Información y Comunicación. Docente en educación para adultos en el programa PILARES de la Ciudad de México. adriana.bravol@educatic.unam.mx
\end{abstract}

1 El trabajo que se presenta forma parte de la tesis realizada por Adriana Bravo para obtener la Licenciatura en Pedagogía, bajo la dirección de la Dra. Kriscautzky en la Facultad de Filosofía y Letras de la UNAM. 


\section{ALFABETIZACIÓN DE ADULTOS DESDE UNA PERSPECTIVA PSICOGENÉTICA}

En este trabajo presentamos algunos resultados de investigación acerca de las hipótesis que elaboran adultos no alfabetizados en torno al sistema de escritura, sus semejanzas y diferencias con respecto al proceso estudiado por Ferreiro y Teberosky (1979) con niños y las implicaciones que resultan para pensar la alfabetización de adultos desde una perspectiva psicogenética. Obtuvimos producciones de adultos mayores en momentos previos a la concepción alfabética del sistema de escritura a través de la presentación de tareas de escritura y de lectura semejantes a las utilizadas en la investigación con niños. Estas producciones nos permiten concluir que existen semejanzas importantes entre niños y adultos en el proceso de construcción de conocimientos acerca del sistema de escritura, aunque también constatamos algunas diferencias relacionadas sobre todo con el tiempo que requiere el proceso de construcción y con la posibilidad de los adultos de insertarse en prácticas sociales del lenguaje escrito. Estos resultados ponen de manifiesto la necesidad de tener en cuenta el pensamiento del adulto a la hora de proponer campañas de alfabetización.

Palavras-chave: Alfabetización. Adultos. Teoría Psicogenética.

\section{ALFABETIZAÇÃO DE ADULTOS EM UMA PERSPECTIVA PSICOGENÉTICA}

Neste trabalho apresentamos alguns resultados de uma pesquisa sobre as hipóteses que adultos não alfabetizados elaboram à respeito do sistema de escrita, suas semelhanças e diferenças quanto ao processo estudado por Ferreiro e Teberosky (1979) com crianças e as implicações decorrentes em pensar sobre o alfabetização de adultos sob uma perspectiva psicogenética. Obtivemos produções de idosos em momentos anteriores à concepção alfabética do sistema de escrita por meio da apresentação de tarefas de escrita e leitura semelhantes às utilizadas em pesquisas com crianças. Essas produções permitem concluir que existem importantes semelhanças entre crianças e adultos no processo de construção do conhecimento sobre o sistema de escrita, embora também encontremos algumas diferenças relacionadas principalmente ao tempo necessário para o processo de construção e à possibilidade de adultos para serem inseridos nas práticas sociais da linguagem escrita. Esses resultados destacam a necessidade de levar em consideração o pensamento do adulto ao propor campanhas de alfabetização.

Palabras clave: Formación inicial. Educación continua. Tecnologías digitales. Necesidades educativas específicas. Accesibilidad.

\section{plurais}

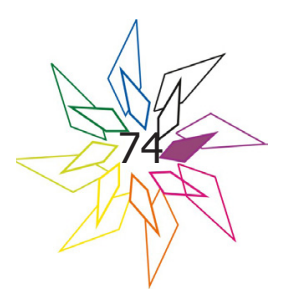




\section{ADULT LITERACY FROM A PSYCHOGENETIC PERSPECTIVE}

In this work we present some research results about the hypotheses that non-literate adults elaborate around the writing system, their similarities and differences with respect to the process studied by Ferreiro and Teberosky (1979) with children and the implications that result in thinking about the adult literacy from a psychogenetic perspective. We work with writing and reading tasks that were similar to those used by Ferreiro and Teberosky in their research with children. We obtained writing and reading evidence from adults in the previous period of alphabetical conception of the writing system. These productions allow us to conclude that there are important similarities between children and adults' process of building knowledge about the writing system, although we also find some differences related especially to the time required for the construction process and to the possibility of adults to be inserted in social practices of written language. These results highlight the need to take adult thinking into account when proposing literacy campaigns.

Keywords: Literacy. Adults. Psychogenetic Theory. 


\section{ALFABETIZACIÓN DE ADULTOS DESDE UNA PERSPECTIVA PSICOGENÉTICA}

\section{Introducción}

Las propuestas de alfabetización de adultos han estado, y están, construidas sobre una visión compartida acerca del sujeto que aprende como un sujeto que debe pasar de "no saber" a "saber leer y escribir" a través de un entrenamiento (INEA, 2015). Aunque existe una diversidad de métodos, la mayoría se sustenta sobre premisas comunes: el proceso de apropiación de la lengua escrita es más rápido en los adultos, es más fácil para los adultos aprender a leer y escribir ya que tienen mayores experiencias de vida que los niños, para ser alfabetizador no se requiere más que una breve instrucción para enseñar y cualquier persona que sepa leer y escribir puede alfabetizar. Como señala Ferreiro:

...todas las propuestas de alfabetización nos parecen insuficientes (independientemente del éxito que se les atribuya), porque toman un punto de partida definido con toda independencia del sujeto de aprendizaje. Ya sea que se decida por las letras, las sílabas, la palabra generadora o los enunciados, esa decisión se toma "desde afuera", sin saber cómo conceptualiza el adulto analfabeto el sistema de escritura socialmente constituido. (Ferreiro, 2007: 21)

En ese sentido, más allá de las diferencias de método, las propuestas de alfabetización se basan en actividades desvinculadas unas de otras, que dosifican el aprendizaje de la lengua escrita decidiendo lo que es más fácil o difícil de aprender desde el punto de vista del adulto alfabetizado. A continuación se muestran algunos ejemplos de las fichas didácticas que se utilizan para la alfabetización.

\section{plurais}


Figura 1. Páginas del libro La palabra

\section{g) Agrupación de palabras por tema}

papa, apio

palo, púa, polea, pala, pule, apalea

- Integración y producción de textos significativos

1. Lupe pela la papa

2. Oí la pelea

3. Polo apila palos

4. El papá pule el palo

\section{g) Familias de palabras}

paño, pañuelo, pañoleta, pañal, apañar

ala, aleta, aletear, alado, alerón

niño, niñez, aniñado

h) Agrupación de palabras por temas

piña, pepita, paleta, atole, totopo,

paté, ate, elote, lata, papa, apio, apetito poeta, piloto

pie, puño, pata, ala

pelota, papalote

Logros de la persona joven y adulta: Reconoce la función de la " $y$ " y la utiliza para formar enunciados.

Materiales: Imágenes, cartones, familias silábicas y palabra familia.

Habilidad ejercida: Comunicación oral, comprensión y producción de textos escritos.

- Temas sugeridos para el diálogo:

La vida familiar. Las relaciones entre padres e hijos. La importancia de ser padres. La planificación familiar. Los hermanos. La posición de cada quien en la familia. Lo que hace cada quien (estudiar, trabajar, en qué). Los problemas familiares. La rutina diaria. La familia y los amigos. ¿Hay amigos que son como de la familia? Amigos, enemigos, familia.

- Separación de palabras en sílabas:

Lee lentamente la palabra familia y pregunta cuántas partes la componen. Después escribe debajo de la palabra las sílabas que la componen:

$$
\text { fa mi lia }
$$

- Familias silábicas que se derivan de la palabra:

familia

\begin{tabular}{c|c|c|c}
\hline $\mathrm{fa}$ & $\mathrm{mi}$ & lia & \\
\hline $\mathrm{fe}$ & $\mathrm{ma}$ & lio & \\
\hline $\mathrm{fi}$ & $\mathrm{me}$ & lie & \\
\hline $\mathrm{fo}$ & $\mathrm{mu}$ & & au \\
\hline $\mathrm{fu}$ & $\mathrm{mo}$ & & oi \\
\hline
\end{tabular}

Recuerda sumar las sílabas de la palabra anterior.

Dibuja en el pizarrón a un señor, una señora y a dos niños o pega una imagen y escribe:

la familia, el papá, la mamá, Toño y Lupe

Pregunta: “¿Qué dice aquí?”.

Fuente: Instituto Nacional para la Educación de adultos (INEA, 2015), México.

Como se puede observar las actividades que se proponen no tienen en cuenta los conocimientos de los sujetos que aprenden, al menos no en tanto sujetos que piensan y construyen conocimiento. En estos métodos se declara tener en cuenta sus saberes, su experiencia de vida e 
incluso sus intereses y necesidades. Pero discurso y metodología no van de la mano. Lo que se dice respetar en el otro desaparece en el momento de plantear actividades sin sentido que no toman en cuenta el proceso de pensamiento de las personas:

El respeto hacia la persona analfabeta no deja de ser un enunciado vacío cuando no sabemos qué es lo que habría que respetar. Conocer al adulto, para que el respeto hacia él sea también un respeto intelectual, nos parece esencial para guiar cualquier acción pedagógica que intente construir a partir de lo que el sujeto ya haya construido por sí mismo, antes de esa acción. (Ferreiro, 2007: 21)

Las investigaciones sobre el proceso de adquisición del sistema de escritura en adultos no alfabetizados (Ferreiro, 2007; Kurlat y Perelman, 2013) no son exhaustivas pero son suficientes para pensar en que es imprescindible plantear una metodología coherente con la base epistemológica que propone la teoría psicogenética a la hora de llevar a cabo una propuesta de alfabetización de adultos.

En este artículo vamos a compartir algunos resultados de investigación acerca de las hipótesis que elaboran adultos en proceso de alfabetización en torno al sistema de escritura con la convicción de que aportar más datos en este campo ayudará a dar fundamento a nuevas propuestas metodológicas que nos ayuden a respetar intelectualmente a los adultos que aprenden a leer y escribir.

\section{Marco teórico}

Como se mencionó más arriba, las investigaciones acerca del proceso de construcción de conocimientos acerca de la lengua escrita con adultos desde la perspectiva psicogenética son escasas. El principal antecedente de nuestro trabajo es el realizado por Ferreiro en 1983 con una muestra de 58 personas entre 17 y 66 años de edad (Ferreiro, 2007). Esa investigación se orientó a partir de los siguientes interrogantes: ¿qué similitudes habrá entre las conceptualizaciones de niños y adultos? ¿habrá niveles de conceptualización ausentes en los adultos? ¿qué diferencias habrá entre los adultos? A partir de estos planteamientos se prepararon situaciones similares a las propuestas en las investigaciones con niños y algunas nuevas, para abordar aspectos de la conceptualización sobre el sistema de escritura y la función de los textos en los contextos propios de los adultos (Ferreiro, 2007).

\section{plurais}

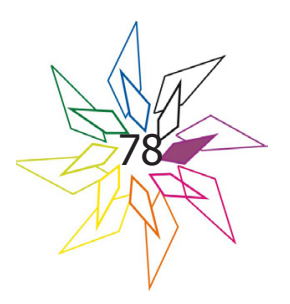


Entre las principales situaciones planteadas están el trabajo de clasificación de material gráfico, interpretación de partes de una oración escrita, escritura e interpretación del nombre propio, identificación de letras y números e interpretación de textos en contextos reales (Ferreiro, 2007).

A través de las entrevistas clínico-críticas retomadas por Ferreiro de su trabajo con Piaget, se plantearon estas situaciones a los 58 adultos y se analizaron de forma rigurosa tanto sus producciones escritas como las interpretaciones de los textos presentados.

Entre los principales resultados destacan:

Las semejanzas son notables en muchos aspectos cuya relevancia teórica no es nada despreciable: los adultos manifiestan los mismos requerimientos de cantidad mínima y de variedad interna que ya conocemos en los niños; manifiestan similar distinción entre "lo que está escrito" y "lo que se puede leer", dificultades similares para manejar las relaciones entre el todo y las partes al nivel de la palabra escrita; etc. pero también es cierto que no todos los niveles de conceptualización identificados en los niños se reencuentran en los adultos: los niveles más primitivos están prácticamente ausentes. (FERREIRO, 2007, p. 205-206)

El trabajo de Ferreiro dejó planteada la importancia de tomar en cuenta lo que saben los adultos en proceso de alfabetización. Apuntó los elementos fundamentales para repensar las acciones alfabetizadoras. Sin embargo, más de 30 años después, la práctica alfabetizadora continúa pensada desde fuera, desde lo que los alfabetizados consideran que es fácil o difícil para los no alfabetizados y desde lo que, políticamente, conviene para no invertir lo que se requiere si realmente se quiere ofrecer oportunidades de inclusión y respeto a los derechos de las personas más vulnerables.

\section{Contexto de investigación}

Las intervenciones realizadas con adultos desde el campo de la investigación se enfrentan a varias dificultades: por un lado, la falta de espacios formales donde asistan adultos de forma regular, el tiempo de asistencia a estos lugares y, por otro, las experiencias negativas por las que han pasado los adultos que inhiben sus respuestas ante preguntas de un diseño de investigación similar al que se ha realizado con niños.

\section{plurais}


Por estas razones, nuestra investigación se realizó en el marco de un taller de alfabetización de adultos (Alf@bit) organizado por la Coordinación de Tecnologías para la Educación (CTE)². El taller fue ofrecido para que los adultos involucrados obtuvieran un beneficio y no participaran solamente como "sujetos de estudio" en una investigación.

La implementación del proyecto Alf@bit nos permitió acceder al pensamiento de los adultos sobre el sistema de escritura en un contexto de beneficio mutuo. A lo largo del taller fuimos implementando algunas situaciones de indagación con tareas similares a las que Ferreiro (2007) diseñó en el trabajo referido con adultos:

- Escritura e interpretación de palabras.

- Escritura e interpretación de oraciones.

- Escritura e interpretación del nombre propio.

Estas tareas, como señalamos, fueron similares pero no idénticas a las presentadas por Ferreiro. La escritura de palabras y oraciones se realizó en el contexto de la escritura de una receta. El trabajo con el nombre propio a través de múltiples situaciones de identificación, lectura y escritura del nombre a propósito de situaciones concretas: saber quién estaba presente en la clase, escribir el nombre para identificar un libro o simplemente "jugar a escribir" nombres y palabras que empiezan igual. Utilizamos diferentes herramientas de escritura: lápiz y papel, teclado y pantalla y fichas de madera con letras. Esto, con la intención de facilitar las tareas cuando el trazado de las letras era complicado en términos motrices y de facilitar la revisión y reescritura ya que a las señoras les causaba conflicto tachar sus producciones.

\section{Metodología de investigación}

Nuestra investigación parte de la hipótesis general de que el proceso de apropiación de la lengua escrita de los adultos es similar al de los niños, tomando como base las investigaciones de Ferreiro desde la perspectiva psicogenética. En consecuencia, la forma de acceder a las hipótesis o al pensamiento de los adultos con respecto a cómo funciona el sistema de escritura es a través

2 La CTE pertenece a la Dirección General de Cómputo y de Tecnologías de Información y Comunicación de la Universidad Nacional Autónoma de México. Dicha Coordinación tiene, entre otros, un proyecto de alfabetización con tecnología e inclusión digital para población vulnerable. Alf@bit es el resultado del trabajo con TIC para promover la alfabetización de adultos trabajando simultáneamente por achicar la brecha digital.

\section{plurais}


de una interacción directa entre el entrevistado y el entrevistador, interacción en la cual se considera al entrevistado como un interlocutor que piensa y cuyas ideas tienen un fundamento que es responsabilidad del entrevistador interpretar y sistematizar. No se trata de observar al adulto ni de tomar sus producciones escritas como evidencias aisladas, sino de entablar un diálogo en el que ambos, entrevistado y entrevistador intercambian ideas en torno al funcionamiento de la escritura a partir de lo escrito y de la interpretación del mismo. Esto es lo que Piaget planteó como entrevistas clínico-críticas, que fueron retomadas por Ferreiro y Teberosky (1979) para su trabajo específico en torno a la construcción del conocimiento sobre el sistema de escritura.

Si bien el contexto de trabajo fue un taller con propósitos de enseñanza, las producciones que reportamos fueron realizadas por las señoras en una situación de entrevista individual con el propósito de acceder a su forma de conceptualizar el sistema de escritura. En esos momentos el trabajo con el grupo fue coordinado por un alfabetizador para dar oportunidad a la entrevistadora de trabajar de forma individual con la entrevistada. Las actividades propuestas se desarrollaron a través del diálogo y la solución de tareas. Nuestras intervenciones no se orientaron a validar respuestas correctas o incorrectas sino que buscaron formular explicaciones plausibles acerca de las respuestas y acciones del entrevistado que se ponen a prueba a través de cuestionamientos, nuevas preguntas o nuevos problemas que los adultos deben resolver.

La población fue fluctuante pero tuvimos la oportunidad de trabajar con 7 señoras entre 57 y 67 años durante un año. Por este motivo algunos de los ejemplos son de las mismas señoras en diferentes momentos del proceso de adquisición del sistema de escritura.

\section{Resultados}

Las mayores coincidencias entre el proceso de adquisición de la lengua escrita de niños y adultos las encontramos durante el periodo que Ferreiro denominó fonetización de la escritura, que inicia con el periodo silábico y culmina con el periodo alfabético. En este sentido, encontramos coincidencias importantes con lo reportado por Ferreiro en 2007. Sin embargo, también encontramos algunos ejemplos de escrituras y problemas anteriores al periodo de fonetización que nos permiten pensar que los adultos siguen un camino similar al de los niños durante todo el proceso.

\section{plurais}




\section{Conflictos con el nombre propio}

Mucho antes de iniciar la educación formal tanto niños como adultos están expuestos a información sobre el sistema de escritura, desde cómo se escribe mamá y papá hasta la escritura correcta del nombre propio. Toda esta información que reciben externamente la modifican para poder transformarla en conocimiento que les permita entender el mundo escrito.

La mayoría de las veces la información que reciben del medio choca con la reflexión propia que hacen sobre el sistema de escritura. Un ejemplo son los conflictos que deben enfrentar en torno a la escritura del nombre propio.

Desde el punto de vista de un adulto alfabetizado la escritura correcta del nombre propio es tomada como una evidencia de que los adultos saben leer y escribir, es por esto que es de las primeras cosas que se enseñan. Sin embargo, los estudios desarrollados por Ferreiro así como el ejemplo que presentaremos a continuación, muestran que la escritura del nombre propio representa grandes desafíos desde el punto de vista del aprendizaje.

En el siguiente caso se muestra el análisis del nombre propio que hace Rosalía, a partir de una actividad que consiste en ordenar y escribir su nombre completo. En este momento del proceso ella sabe identificar su nombre dentro de un conjunto de nombres. También sabe de memoria cuáles son las letras con las que se escribe su nombre. Para esta actividad se le dan a Rosalía unas fichas de madera con el alfabeto, cada ficha tiene una letra escrita. A ella se le dan todas las letras que corresponden a la escritura de su nombre completo: Rosalía Acolman Martínez, separadas en tres grupos de letras. Posteriormente, se le da la indicación de ordenar todas las letras para que quede escrito su nombre completo, sin que le sobre ninguna letra.

Rosalía hace esta actividad mecánicamente, llevando el orden en las letras que le han enseñado para la escritura de su nombre y haciendo la separación de su nombre y sus apellidos ${ }^{3}$. El problema surge cuando le pedimos la lectura:

3 Por respeto a sus datos personales modificamos los apellidos, respondiendo a las mismas características de los originales. El primer apellido se compone de tres sílabas de tipo V-CVC-CV iniciando con la letra A y una L en la segunda sílaba. El segundo conserva la estructura de las sílabas y comienza con M.

\section{plurais}

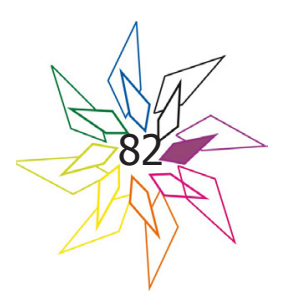




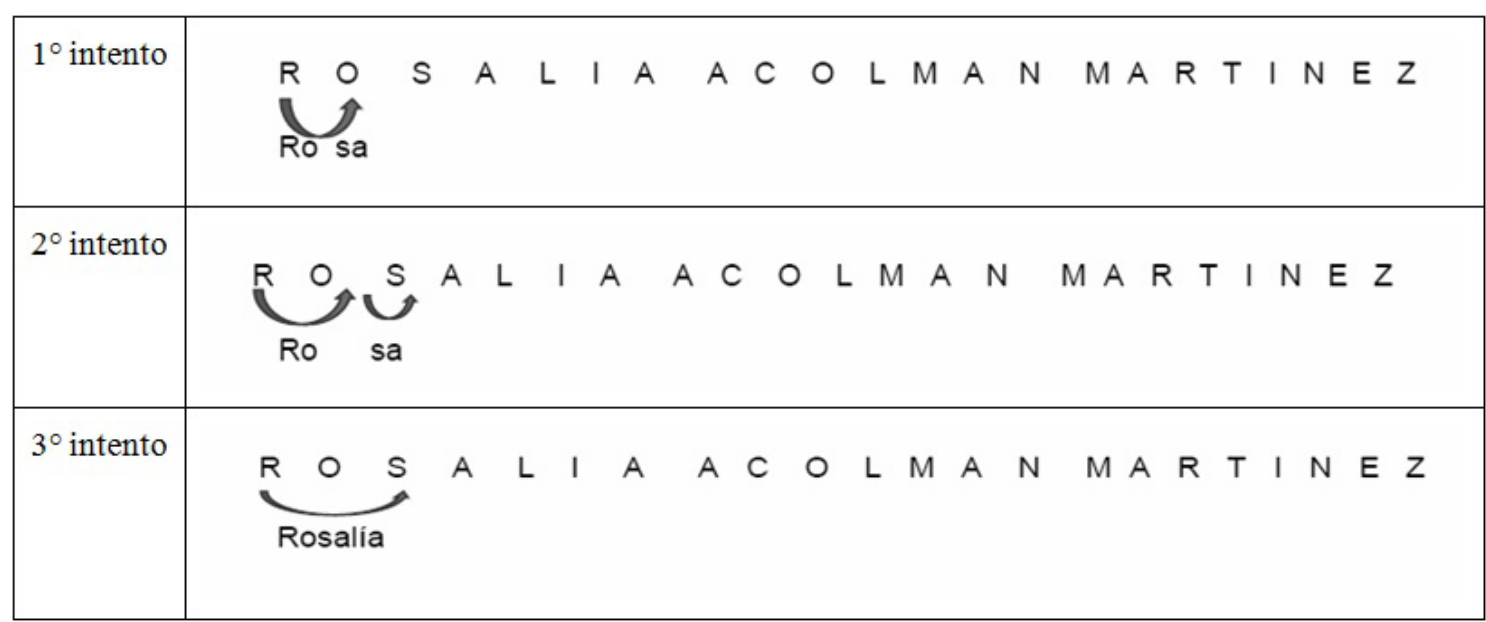

Rosalía duda y se pone nerviosa porque ve que aún le faltan muchas letras por leer, le ayudamos mostrándole los tres grupos de palabras y preguntando dónde dice su nombre y dónde sus apellidos.

R: Ah sí, sí es cierto (Le pedimos que trate de leerlo con calma. Rosalía lee de nuevo)

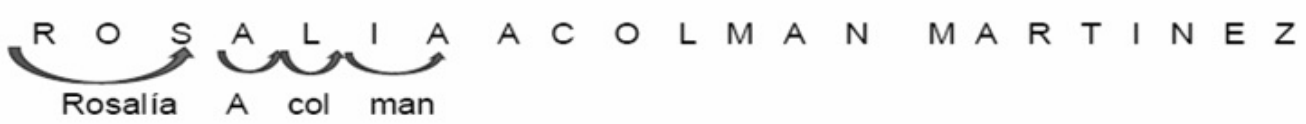

Rosalía es capaz de ordenar las letras de su nombre pero todavía no comprende por qué se necesitan todas esas letras para escribirlo. La solución que plantea resulta bastante conveniente porque fonéticamente la A coincide con el inicio de su primer apellido, y la L queda bien con la sílaba col, estas coincidencias le permiten pensar que con sólo esas letras puede escribir su nombre y su primer apellido. Es importante señalar que al momento de realizar esta interpretación de su nombre Rosalía todavía no podía escribir por sí misma ya que siempre utilizaba sílabas y las combinaba sin lograr entender lo que hacía (Rosalía había estado dos años acudiendo al círculo de estudio del INEA trabajando con palabras generadoras y familias silábicas).

\section{plurais}




\section{La construcción de la hipótesis silábica}

La hipótesis silábica, con la que inicia el tercer periodo que Ferreiro denominó fonetización de la escritura, es muy importante en el proceso por dos razones: "permite tener un criterio general para regular las variaciones en la cantidad de letras que deben escribirse, y centra la atención del niño sobre las variaciones sonoras entre las palabras" (Ferreiro, 1998, pág. 20). En nuestra experiencia encontramos múltiples ejemplos de esta interpretación del funcionamiento del sistema de escritura. Veamos uno, a partir de la situación de escritura de palabras en el contexto de una nota de compra del mercado, Josefina escribe lee y escribe de la siguiente manera:

\begin{tabular}{|c|}
\hline $\begin{array}{c}\text { C H A Y O T E } \Rightarrow \text { C O T } \\
\text { cha yo te }\end{array}$ \\
\hline E S P I N A C A S $\Rightarrow$ E I A S \\
es pi na cas
\end{tabular}

Para leer chayote no tiene problemas: una letra para cada sílaba, tal como se ha reportado en muchas ocasiones con niños:
"Al escribir, el niño trata de encontrar las unidades sonoras que correspondan a las letras y para ello hace uso de sus conocimientos sobre los enunciados orales. Las unidades pronunciables que descubre son las sílabas, repitiendo el nombre que tiene que escribir varias veces para sí mismo" (FERREIRO \& TEBEROSKY, 2000, p. 8)

Sin embargo, al leer espinacas no queda conforme con su lectura, repite cas dos veces en voz baja para sí misma, dudando si le hacía falta una letra más, por esta razón hace una segunda lectura donde utiliza la A para dar lectura a la sílaba $n a$, y luego vuelve a usar la misma A junto con la S para leer cas, de esta forma queda conforme con su escritura.

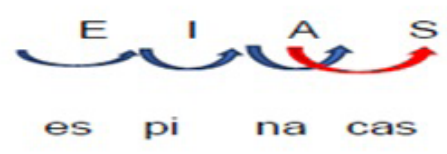


La escritura que hace Josefina responde a un recorte silábico: cada sílaba de la palabra representada con una letra, aunque no de un modo estricto ya que la $\mathrm{S}$ no le resulta suficiente para representar la sílaba cas y recurre a la A interpretada para la sílaba anterior.

Uno de los conflictos que se enfrentan durante el periodo silábico aparece a la hora de interpretar escrituras, especialmente cuando las escrituras quedaron iguales para palabras diferentes. Veamos un ejemplo. Lupita es capaz de escribir su nombre completo de forma convencional. Sin embargo, cuando escribe otras palabras lo hace de forma silábica y, en palabras bisílabas, comienza a completar alguna de las sílabas. En una de las actividades de escritura de palabras se le pide que escriba la palabra musa porque antes escribió esa (mesa). Lupita escribe USA y de inmediato le pedimos que escribiera rusa con el siguiente resultado:

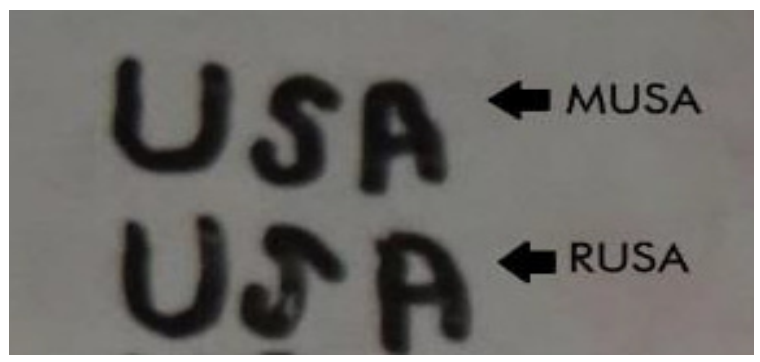

Escritura de musa y rusa

\begin{tabular}{|l|}
\hline Mientras escribe $m u s a$ Lupita lee $m u$ cuando pone la U y sa cuando termina de escribir SA. \\
\hline Cuando escribe rusa Lupita dice: —ru-sa.. ru, otra vez la U. \\
Lee $r u$ y decide poner la S mientras dice $s a$, mira su escritura de musa y nota que le están que- \\
dando iguales. \\
Intenta leer $m u s a$ para sí misma señalando la primera palabra. \\
\hline Vuelve a su escritura de rusa y la lee mostrando lo que está escribiendo. \\
Dudosa decide poner una A al final para concluir la escritura de la palabra rusa que queda así: USA \\
\hline
\end{tabular}


Después de que Lupita termina de escribir el entrevistador interviene:

E: pero si aquí dice musa (señalando la primera palabra) y aquí dice rusa (señalando la segunda palabra) yo las veo iguales ¿cómo es eso posible?

L: aah eso sí verdad... aquí dice rusa y aquí dice musa (señalando confundida)

E: ¿son iguales?

L: no (duda)

E: ¿qué le faltará? Para que en uno diga musa y en otra rusa

L: a ver... aquí dice musa (señalando la primera palabra) y aquí tiene que decir rusa (señalando la segunda palabra)

E: ajá...pero son iguales diría lo mismo si están iguales... entonces para que una diga musa y en otra rusa...

L: (interrumpe) ¡le falta una letra!

En este momento Lupita identifica el problema- “iLe falta una letra!”- pero no puede resolverlo. Reconoce que dos palabras distintas deben estar escritas de forma distinta y se necesita más de una letra para representar una sílaba.

Para ayudarla a resolver el problema escribimos en otra hoja ruta indicando que se fijara qué letras le podían servir para escribir rusa. De esta forma Lupita logró hacer una diferenciación entre ambas palabras, dejando musa como USA y rusa escrita de forma convencional.

\section{Desorden con pertinencia}

Entre el periodo silábico y el alfabético se produce un nuevo análisis sobre el eje cuantitativo y cualitativo de la escritura, éste surge a partir de las dificultades para mantener estable la hipótesis de cantidad mínima junto con la hipótesis silábica.

La sílaba oral es considerada desde dos anclajes diferentes. Las letras elegidas corresponden a esos dos anclajes. A una centración en el "lado vocálico" de la sílaba sucede luego una centración en el "lado consonántico" de la misma sílaba. La misma sílaba es escuchada "desde otro lugar". Escuchada y "vista" porque la escritura permite verla. (Ferreiro, 2009, pág. 3)

\section{plurais}


Para ejemplificar el desorden con pertinencia retomaremos las producciones de Josefina durante la actividad de escritura de palabras. Josefina tenía que escribir el nombre de algunas frutas. Para ello primero debía pronunciarlas y decidir si empezaban o no igual.

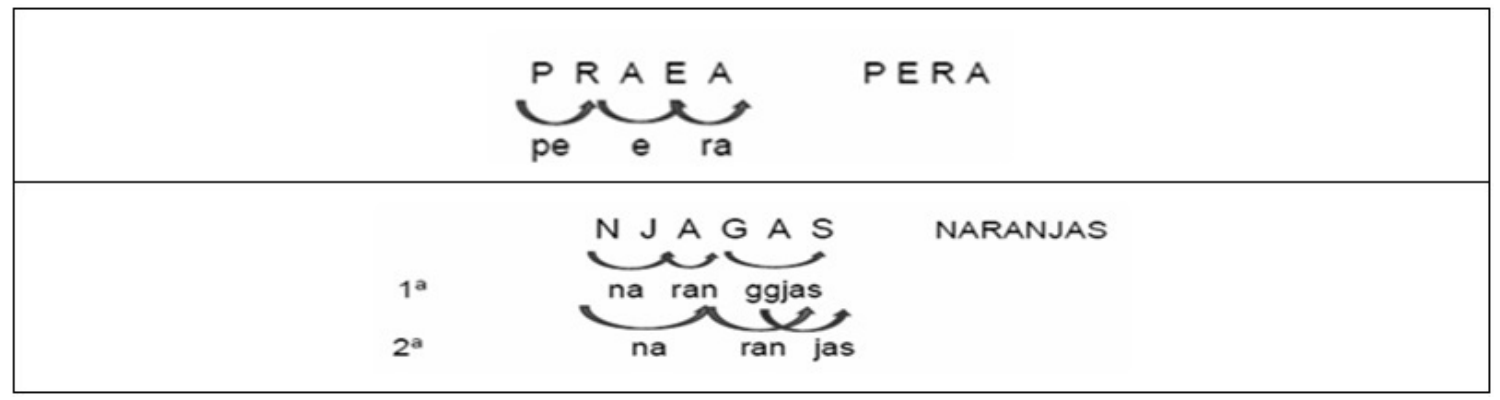

En el primer ejemplo Josefina pone inicialmente PRA, hace una pausa y continúa poniendo la E seguida de la A mientras dice en voz baja pe - ra. En este momento puede evidenciarse otro análisis fonético de la palabra porque las siguientes dos letras que decide poner, la $\mathrm{E}$ y la $\mathrm{A}$, corresponden de nuevo a la escritura de pera, ahora pensada y escuchada desde otro anclaje. Cuando le pedimos que leyera cómo dice pera, ella trató de agrupar las letras de modo que tuvieran sentido. Sin embargo, no quedó convencida con su lectura.

En el ejemplo de la palabra naranjas, Josefina hace la misma pausa después de haber escrito NJA y dice: na-ran-jas... jas...jjjas. Y continúa escribiendo G A S. Lo que sucede en este momento es que Josefina vuelve a pensar en la escritura de la palabra, pero sólo en el final. Sin embargo, como ya había escrito una $J$ la solución es poner ahora una $G$ para intentar dar el sonido final. Cuando le pedimos la lectura, Josefina tiene varias dificultades para darle sentido a todas las letras que puso, primero trata de agruparlas de una forma pero no queda convencida al leer $n a$ sólo con la $\mathrm{N}$ y la J de modo que en su segunda lectura decide integrar las primeras tres letras para $n a$; para leer ran utiliza la $\mathrm{G}$ y la $\mathrm{A}$, pero no queda muy convencida porque al final sólo le quedaría la $\mathrm{S}$ para leer jas y en este momento ella reconoce que una letra no basta para leer, de modo que vuelve a utilizar la A que uso para la sílaba anterior y lee jas aunque no queda conforme con su lectura. 


\section{Escritura de oraciones en contextos reales}

Antonia puede escribir algunas palabras de forma convencional y otras con algunas sílabas completas y otras no (particularmente cuando se trata de sílabas complejas de estructura CCV o CVC). Durante la tarea de escritura de oraciones, en el contexto de escribir una receta para la elaboración de un flan, escribe:

\begin{tabular}{|c|c|}
\hline 2latacvl & dos latas de clavel \\
\hline AUKAVIANLLA & azúcar y vainilla \\
\hline EFIRCAMOL & freír el caramelo \\
\hline
\end{tabular}

Antonia, como muchos de los niños reportados en investigaciones de esta línea teórica, escribe sin separar palabras y usa indistintamente mayúsculas y minúsculas. No representa artículos ni conectores, sólo escribe verbos y sustantivos. Podemos observar algunas palabras escritas de forma convencional, como lata, y otras de forma silábica, como $c v l$ (clavel), así como escrituras intermedias o silábico-alfabéticas tales como AUKA (azúcar), VIANLLA (vainilla) o CAMOL (caramelo). También es de destacar que con frecuencia invierte el orden de las letras para realizar un recorte silábico en sílabas simples cuando aparece un diptongo, como en el caso de VIANLLA, que es más sencillo de leer (VI-A-N-LLA) que cuando aparece el diptongo VAI.

\section{El uso del comodín}

Las reflexiones que hacen los niños sobre la relación entre lo que se lee y lo que se escribe se da en distintos niveles. Una de las reflexiones es en torno a los nombres de las letras, "los niños piensan que si existen letras consonantes con nombres silábicos (la ka, la pe, be) deben existir nombres silábicos que se corresponden con letras consonánticas" (Sciurano, 1997, pág. 39). De este modo los niños creen que existe la "ma" la "ta" la "pa" y que pueden representarse con una letra, por lo que tratan de utilizar letras que conocen para asignar los significados silábicos faltantes dentro de sus escrituras.

Las letras que utilizan llegan a servir de comodines que "son un caso particular del uso de letras en función de sustituta, porque las colocan para sustituir a una letra que están seguros debería ir en la palabra escrita, pero que no saben cuál es" (Sciurano, 1997, pág. 39). De este modo una

\section{plurais}


misma letra puede tener distintos valores sonoros en varias escrituras; una $\mathrm{N}$ puede ser la $z a$ de manzana o la pa de papaya.

La creencia de que existe una letra que corresponde a un sonido silábico, (que existe una "te, un "me"), muchas veces lleva a los niños a incorporar letras consonánticas que conocen sólo por su forma. En estos casos los niños no tienen la certeza de haber colocado la letra adecuada, pero de todas maneras las utilizan y las mismas pasan a funcionar como comodines silábicos. (SCIURANO, 1997, p. 39)

Pudimos identificar el uso del comodín en las escrituras de nuestro grupo, especialmente en la transición a la hipótesis alfabética. Veamos algunos ejemplos de las escrituras de Josefina en las cuales utiliza la $\mathrm{N}$ como un comodín tanto para completar una sílaba como para representar una sílaba simple:

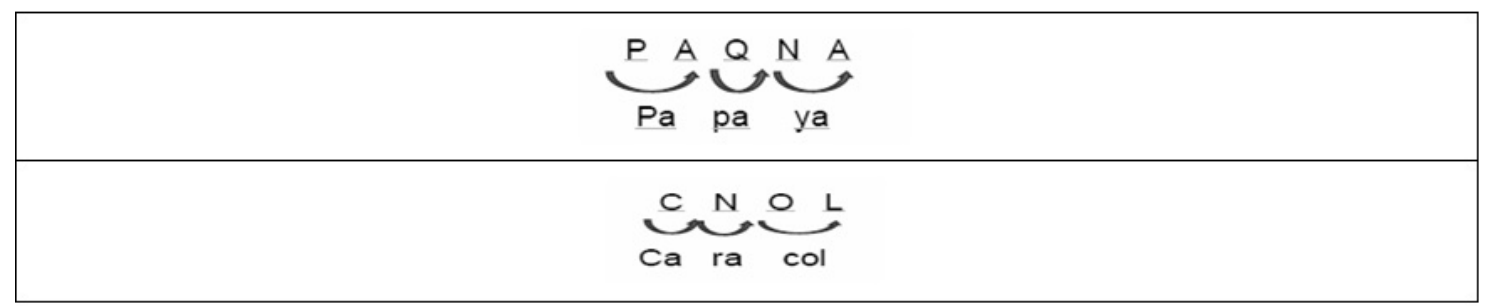

En este caso, la $\mathrm{N}$ aparece para completar la sílaba ya en papaya, y para representar la sílaba ra en caracol.

La letra Q representa la sílaba $p a$ y sirve para diferenciar dos sílabas iguales $p a$ y $p a$.

En el caso de manzanas, recurre nuevamente a la Q como comodín, con una función muy semejante a la que describimos en papaya:

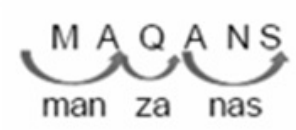


Como se puede observar, la letra Q es usada como comodín para poder nombrar la sílaba "za", cabe resaltar que manzanas es una palabra que "lleva muchas A" por lo que el conflicto es comprensible si pensamos en la hipótesis de variedad interna. Mientras Josefina escribe, va diciendo para sí: man-za-nas... za... Decide escribir la Q para representar $z a$ pero hace una mueca de descontento porque sabe que la $\mathrm{Q}$ no corresponde a la escritura de manzanas pero es la solución que encuentra en este momento del proceso.

\section{Escribir, revisar y completar}

Las siguientes escrituras ejemplifican otros procesos de reflexión por parte de los adultos que fueron propiciados por la escritura en computadora y por la propuesta de revisar lo escrito en una sesión posterior. Las nuevas tecnologías no van a eliminar los problemas conceptuales que deben ser resueltos por el sujeto (niño o adulto). Comprender la naturaleza de un sistema alfabético de escritura utilizando lápiz o papel, o pantalla de computadora y teclado, no es conceptualmente diferente. (Ferreiro, 2007, pág. 314). Sin embargo, la posibilidad de revisar y modificar el texto sin tener que reescribirlo por completo es muy importante en este momento del proceso ya que de otra manera sería agotador volver a escribir todo. Además, como señalamos al inicio, a las señoras participantes les causaba conflicto tachar sus producciones en papel.

La siguiente actividad corresponde a la escritura de una receta de flan:

\begin{tabular}{|l|l|}
\hline \multicolumn{1}{|c|}{ Versión 1 } & \multicolumn{1}{|c|}{ Versión 2} \\
\hline Suevos & 5uevos \\
\hline Ualtadlheeva & Ua Ita de lehe evaora \\
Coesa & Ua talde Codesa \\
Asucar & Asucar \\
Vaina & Vainiya \\
Terolsevo & ponr Iso uevos \\
Toralehe & ponr la lehe \\
TOerelaluadoavaina & TOerer todo al luadoa covaina \\
Seluatobo & Se lua todo \\
Porlascrelste & Por la asucar satn \\
Drilasrcamoerasten & Drilasrcamoerasten \\
Basrlcrmol & Basrlcrmol \\
Vasrenlmellfna & Vasrenlmellfna \\
perevoamialsmons & Perevoamialsmons \\
\hline
\end{tabular}

\section{plurais}

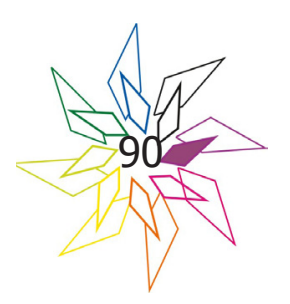


Dos versiones de la receta de flan escrita por Josefina

Transcricpión normalizada de la receta

5 huevos

Una lata de leche evaporada

Condensada (leche condensada en primera lectura) Una lata de condensada (segunda escritura)

\author{
Azúcar \\ Vainilla \\ Poner los huevos \\ Poner la leche \\ Poner en la licuadora la vainilla \\ Se licua todo \\ Poner el azúcar en el sartén \\ Derretir el azúcar y el caramelo en el sartén \\ Vaciar el caramelo \\ Vaciar en el molde el flan \\ Poner en baño María los moldes.
}

En términos de la producción de un tipo de texto Josefina ya sabe que una receta está compuesta por los ingredientes y el procedimiento. Con esta información inicia su texto escribiendo en diferentes líneas cada uno de los ingredientes y luego los pasos a seguir para la elaboración del flan.

En la primera versión de la receta se puede observar que no hay ningún tipo de separación. En una sesión posterior se le invita a revisar su escritura. 
E: Josefina aquí me había dicho que decía una lata de leche evaporada ¿sí dice eso? A ver vamos a leer.

Josefina intenta leer:

J: (deletreando mientras señala con el dedo): u-a...una.../ no...mmm... una ta...// no, me faltó

E: ¿Qué le faltó?

J: Es que está todo junto / me faltaron los espacios

E: ah bueno, a ver vamos a poner los espacios // aquí me dijo que tiene que decir una lata de leche evaporada, vamos a ver hasta dónde dice una y dónde dice lata...

Josefina empieza a poner los espacios por sí misma. Con ayuda de las flechas de la computadora ella recorre el cursor para ponerlo en medio de donde cree que termina una palabra y empieza otra. Cuando termina de poner los espacios se da cuenta de que le quedan segmentos muy pequeños así que cuando intenta leer dice: jahora me faltan letras!

Finalmente, Josefina arregla su escritura poniendo espacios y agregando letras que consideró que le hacían falta, dejando su producción como se muestra en la segunda versión. El avance entre las dos versiones es evidente porque Josefina puede mirar lo escrito y modificarlo sin volver a escribir todo, cosa que es posible gracias al uso del procesador de texto.

\section{Evidencias encontradas sólo con adultos}

Como parte final del análisis de nuestras evidencias describiremos a continuación algunos ejemplos encontrados con adultos que no tienen un análisis comparativo con las investigaciones de Ferreiro hechas con niños. Consideramos que pueden ser relevantes para futuras investigaciones con un mayor nivel de análisis. Los datos se basan en dos aspectos, al primero lo denominamos lectura invertida en palabras que inician con vocal y al segundo segmentación de palabras antes de la hipótesis alfabética de la escritura.

\section{Lectura invertida en palabras que empiezan con vocal}

El primer caso se presentó en distintos momentos durante nuestras intervenciones con Josefina en la lectura de varias palabras. De manera sistemática ella intenta leer las palabras que inician con vocal de manera invertida:

\section{plurais}




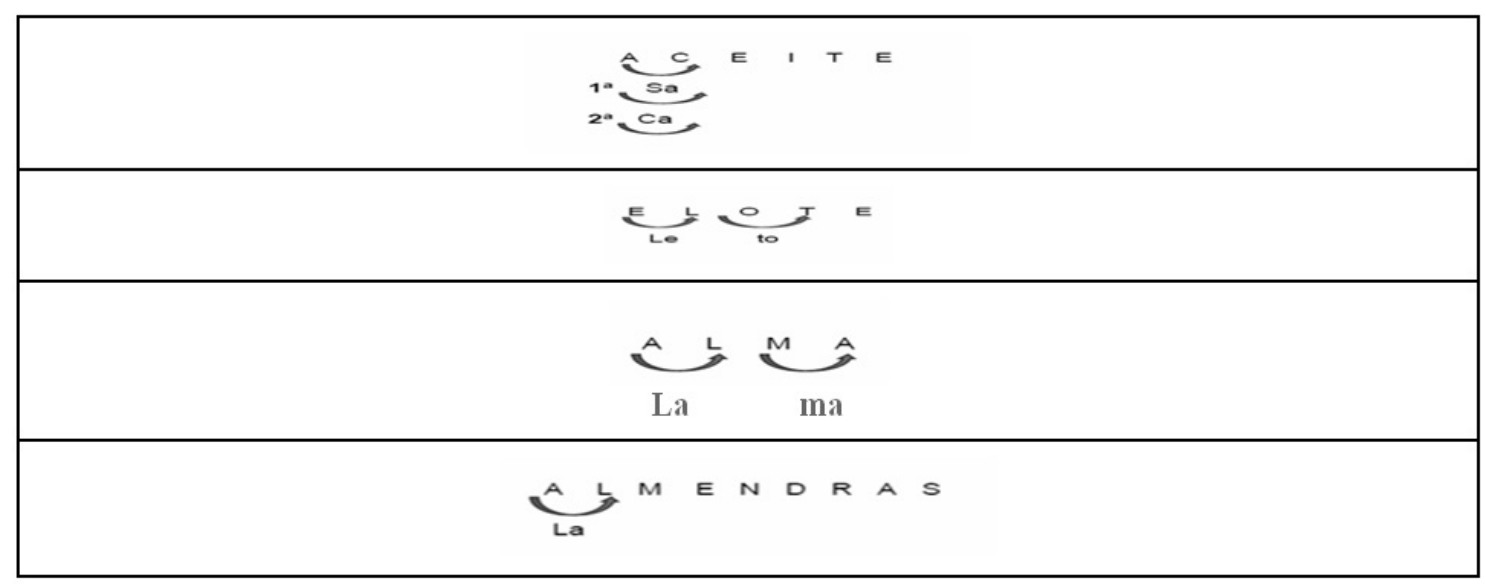

Paradójicamente, aunque con menor frecuencia hay otras palabras que inician con consonante que Josefina invierte en la lectura, por ejemplo:

\begin{tabular}{|l|}
$\underbrace{\text { LA }}_{\text {Al }}{ }^{T}{ }^{\text {S E }}$ \\
\hline
\end{tabular}

Esta característica tan peculiar se presentó también con Antonia. Al pedirle que nos leyera su receta tuvo algunos problemas con la lectura de algunos ingredientes.

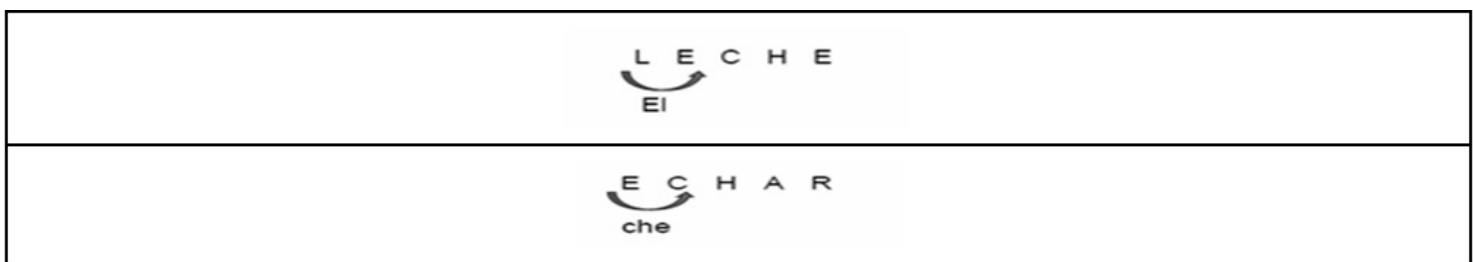

Consideramos que es necesario iniciar futuras investigaciones sobre este aspecto en específico para determinar si es una característica del proceso o es una situación individual. 


\section{Segmentación antes de la hipótesis alfabética}

En el caso de los niños la reflexión sobre la segmentación entre las palabras ocurre generalmente una vez que se ha establecido la hipótesis alfabética de la escritura. Por el contrario, la experiencia con las señoras nos indica que el espacio entre las palabras es una información que obtienen del medio y que incorporan mucho antes de escribir alfabéticamente. Incluso la reflexión sobre esta separación les permite en algunas ocasiones incorporar más letras en la escritura de algunas palabras, logrando así una escritura más cercana a la convencional. Al respecto, en el apartado Escribir, revisar y completar presentamos el caso de Josefina y su receta de flan, en la que al revisar incluyó espacios para segmentar la escritura aún cuando ésta no era alfabética.

Ahora bien, la segmentación no se realiza siempre de forma convencional, apareciendo en algunos casos como hiposegmentaciones y en otro como hipersegmentaciones. En el siguiente ejemplo de Antonia observamos una segmentación que entra en conflicto con la cantidad mínima de letras aceptable para que algo esté escrito:

La siguiente receta fue hecha por Antonia, los fragmentos corresponden a algunos pasos para la elaboración de arroz.

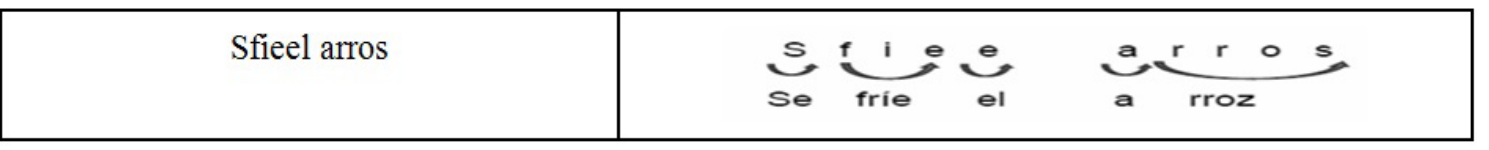

La segmentación de las palabras en esta oración resulta muy compleja desde el punto de vista del adulto en proceso de aprendizaje, dado que segmentar convencionalmente generaría grupos muy pequeños de letras que entran en conflicto con la hipótesis de cantidad mínima. Por esta razón Antonia decide dejar una cadena gráfica para se fríe el y otra para arroz.

Por otra parte, la segmentación puede pasar de ser nula, a suponer que se debe separar a partir del recorte silábico que identifican en la oralidad. Por ejemplo, a Josefina se le pregunta cuántas palabras debe poner para escribir lavar la estufa. Josefina hace un recorte silábico: la-var la es-tu-fa mientras cuenta con los dedos el número de segmentos que identifica. Tras este ejercicio ella responde que debe poner seis palabras. Aunque un poco extrañada comienza a escribir poniendo sólo un espacio entre las letras, sin representar el artículo.

\section{plurais}




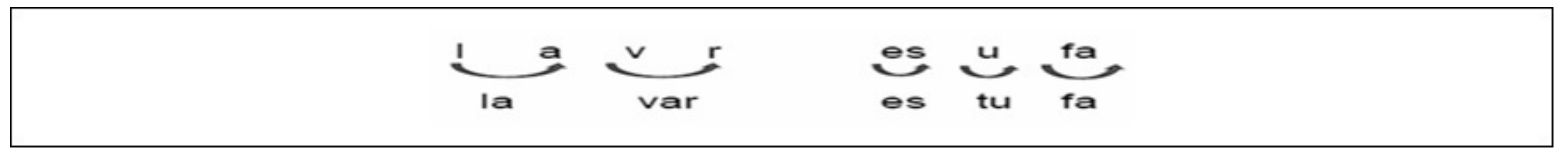

Comprender la función de la segmentación es un proceso que se estabiliza de manera posterior a la hipótesis silábica y silábico-alfabética en los niños. Sin embargo, en los casos explorados con los adultos la reflexión en torno a la segmentación de las palabras se presenta mucho antes de comprender el funcionamiento alfabético del sistema de escritura porque reconocen que sus escrituras no pueden estar "todas pegadas", información que proviene del entorno social y que tiene mucho peso en la producción escrita, mucho más que en el caso de los niños.

\section{Conclusiones}

El análisis de los datos que se obtuvieron a partir de esta investigación nos permite mirar el proceso de alfabetización en adultos como un proceso complejo, que inicia mucho antes de la implementación de las campañas de alfabetización y que en definitiva no puede durar menos de un año y mucho menos los 3 meses que establece una campaña.

A partir del análisis de nuestras evidencias podemos afirmar que el proceso de adquisición de la lengua escrita de niños y adultos sigue un recorrido semejante ya que las hipótesis que elaboran los adultos aparecen en el mismo orden identificado en los niños, así como los conflictos en la interpretación del nombre propio o la dificultad para aceptar escrituras de menos de tres letras o de letras iguales. Durante el periodo silábico la escritura con valor sonoro convencional se presentó en todos los casos que analizamos, haciendo uso de vocales y consonantes en las producciones.

La escritura de textos nos permitió obtener enunciados largos en los cuales se puede apreciar, tal como sucede con los niños, una escritura de un nivel menor que al escribir palabras. Es decir, si al escribir palabras lograban una escritura silábico alfabética o alfabética, al escribir textos regresaban a una representación silábica estricta donde todas las sílabas de la palabra están representadas por una letra, sin que falte ninguna sílaba por representar, incluyendo los bisílabos y monosílabos

Sólo en tres de los siete casos que analizamos también identificamos una característica de las escrituras durante la hipótesis silábica: sólo se escriben las cosas (los sustantivos) para que una oración pueda leerse. Sin embargo, en la interpretación de sus propias escrituras los artículos, preposiciones

\section{plurais}


y conectores sí son nombrados. Es decir, son necesarios para poder entender el mensaje escrito, pero no hace falta escribirlos.

En el periodo silábico alfabético identificamos el desorden con pertinencia en todas las señoras que se encontraban en este nivel; sobre todo en palabras compuestas por sílabas complejas CCV y aquellas que inician con vocal, por ejemplo: aceite, flan, manzana, aceitunas.

Una de las diferencias la encontramos en la segmentación de las palabras; en los estudios realizados y dirigidos por Ferreiro con niños, la segmentación ocurre una vez que se ha consolidado la hipótesis alfabética. Sin embargo, en los casos analizados con personas adultas podemos reconocer que la segmentación es un aspecto que se presenta mucho antes y que incluso permite que puedan acercarse cada vez más a la hipótesis alfabética.

Una distinción más corresponde a la inversión de letras en la lectura de algunas palabras, lo cual no está registrado con niños hasta el momento. En nuestras evidencias podemos apreciar que al pedirles la lectura de algunas palabras, específicamente aquellas que comienzan con vocal, los adultos hacen una inversión pasando de una sílaba constituida por una vocal a una sílaba simple CV. Algunos de estos ejemplos sucedieron con las palabras: atún, aceite, aceituna, atole, elote. Palabras que inician leyendo como " $t a$ ", " $c a$ ", " $c a$ " "te" y "le" respectivamente. Es necesario realizar futuras investigaciones para comprobar la inversión de letras en palabras que inician con vocal, no sólo en la escritura sino también en su lectura.

Aún cuando reconocemos las limitaciones evidentes de nuestra investigación, consideramos que estos resultados pueden contribuir a replantear los métodos que se utilizan para la alfabetización de adultos ya que ponen de manifiesto que las personas en proceso de aprendizaje elaboran hipótesis para tratar de comprender qué es lo que la escritura representa. Además, constatamos que este proceso lleva tiempo y no es posible acelerarlo tal como se supone en las campañas actuales. La presentación de sílabas o fonemas para combinar en palabras contraviene lo que los adultos están pensando, pero es difícil que lo externen porque en general tienen experiencias educativas y de vida poco favorables que les han reforzado la idea de que lo que piensan es "incorrecto".

En suma, si queremos alfabetizar respetando a las personas es imprescindible reconocer su proceso de construcción de conocimiento. El respeto al que tienen derecho no es solamente en relación con sus intereses o historias de vida, sino también (y principalmente) tienen derecho a ser respetados como personas que piensan y no como receptores de información y adiestramiento.

\section{plurais}




\section{REFERENCIAS}

CASTEDO, Mirta, \& MOLINARI, C. Leer y escribir en proyectos. Revista Brasileira de Educação, 16-24, 2003.

FERREIRO, Emilia, \& TEBEROSKY, Ana. Los sistemas de escritura en el desarrollo del niño. México: Siglo XXI, 1979.

FERREIRO, Emilia, \& TEBEROSKY, Ana. La comprensión del sistema de escritura: construcciones originales del niño e información específica de los adultos. Revista Latinoamericana de Lectura (Lectura y vida), 10, 1981.

FERREIRO, Emilia. Alfabetización teoría y práctica. México : Siglo XXI, 1998.

FERREIRO, Emilia; TEBEROSKY, A., CASTORINA, J. A.,GRUNFELD, D; AVENDAÑO, F., \& BAEZ, M. Sistemas de escritura, constructivismo y educación. Argentina: Homo Sapiens, 2000.

FERREIRO, Emilia. Alfabetización de niños y adultos Textos escogidos. Pátzcuaro, Mich., México: CREFAL. (Paideia latinoamericana; 1), 2007.

FERREIRO, Emilia. La desestabilización de las escrituras silábicas: alternancia y desorden con pertinencia. Revista Latinoamericana de Lectura (Lectura y vida), junio, 13, 2009.

INEA. 2015 Paquete del alfabetizador. México. Disponible en: http://www.inea.gob.mx/colaboracion/asuntos_internacionales-240ct2018/documentos/pdf/Paquete_del_alfabetizador_3.pdf. Consultado el 12 de agosto de 2019.

KURLAT, Marcela \& PERELMAN, F. Procesos de alfabetización inicial en personas jóvenes y adultas. ¿Hacia un proceso de inclusión? Revista del IICE /32, pp. 55-72, 2013.

SCIURANO, G. Q. El uso y función de las letras en el periodo pre-alfabético. México: CINVESTAV-IPN, 1997.

Recebido em: 20 de julho de 2020 .

Inserido em: 15 de outubro de 2020.

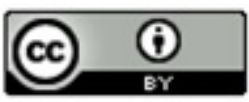

Esta obra está licenciada com uma Licença Creative Commons Atribuição 4.0 Internacional.

\section{plurais}

